\title{
Temperature stability of field induced anisotropy in soft ferromagnetic Fe,Co-based amorphous and nanocomposite ribbons
}

\author{
P. R. Ohodnicki, ${ }^{1,2, a)}$ D. E. Laughlin, ${ }^{1,2}$ M. E. McHenry, ${ }^{1}$ V. Keylin, ${ }^{1,2}$ and J. Huth ${ }^{2}$ \\ ${ }^{1}$ Department of Materials Science and Engineering, Carnegie Mellon University, Pittsburgh, Pennsylvania \\ 15213, USA \\ ${ }^{2}$ Magnetics, Division of Spang and Company, Pittsburgh, Pennsylvania 15238, USA
}

(Presented 13 November 2008; received 30 August 2008; accepted 7 November 2008; published online 11 March 2009)

\begin{abstract}
The temperature stability of field induced uniaxial anisotropy $\left(K_{U}\right)$ was investigated by thermomagnetic treatments of $\left(\mathrm{Co}_{1-x} \mathrm{Fe}_{x}\right)_{89} \mathrm{Zr}_{7} \mathrm{~B}_{4}$ amorphous ribbons after field annealing below and above the crystallization temperature. We conclude: (1) Field annealing treatments are necessary to properly investigate the temperature stability of $K_{U}$, (2) $K_{U}$ of field crystallized alloys exhibit improved temperature stability relative to alloys remaining amorphous after field annealing, and (3) larger $K_{U}$ is obtained for field crystallization treatments as compared to zero-field crystallization followed by field reannealing. Field crystallization may be required for elevated temperature applications when field induced anisotropy is critical for performance. () 2009 American Institute of Physics. [DOI: 10.1063/1.3068547]
\end{abstract}

Magnetic field annealing ${ }^{1,2}$ is used to tailor magnetic properties of soft magnetic materials by inducing a uniaxial magnetic anisotropy energy, $K_{U}$. The induced easy axis can be controlled by applying a saturating magnetic field (i.e., field annealing). However, induced anisotropy due to "selffield annealing" 2 also develops in the absence of an external field resulting in a spatially varying distribution of induced anisotropies that stabilize the remanent domain structure of the material. Field annealing treatments are particularly important for soft ferromagnetic Fe,Co-based amorphous and nanocomposite alloys. The effective magnetocrystalline anisotropy is greatly reduced by exchange coupling of regions with rapid spatial variations (over $\sim 10^{-10}-10^{-9} \mathrm{~m}$ ) of local easy axis orientations. ${ }^{3} K_{U}$ due to induced magnetic anisotropies can dominate the effective magnetocrystalline anisotropy because of greater spatial coherency. ${ }^{4-6}$

Toroidal tape wound cores of amorphous or nanocomposite ribbons can be annealed in a longitudinal or a transverse saturating field. Large $K_{U}$ is often undesirable for zero or longitudinally field annealed cores as it can dominate the effective magnetic anisotropy and result in large hysteretic losses due to domain wall pinning. ${ }^{4}$ However, for transverse field annealed cores large $K_{U}$ can be desirable resulting in higher saturation fields, lower permeabilities, and improved high frequency response, ${ }^{7,8}$

$K_{U}$ in $\mathrm{Fe}$,Co-based soft magnetic amorphous and amorphous/nanocrystalline "nanocomposite" alloy systems has been reported and discussed in a number of previous works. ${ }^{5-12}$ Co-rich nanocomposites tend to exhibit relatively large values of $K_{U}$ as compared to the corresponding Fe-rich compositions. ( $\mathrm{Fe}, \mathrm{Co})-\mathrm{Zr}-\mathrm{B}$ is one of the most widely studied alloy systems due to high saturation magnetizations and Curie temperatures. We have previously reported the compo-

\footnotetext{
${ }^{a)}$ Author to whom correspondence should be addressed. Electronic mailpaul.ohodnicki@gmail.com.
}

sitional dependence of $K_{U}$ for transverse field annealed $\left(\mathrm{Co}_{1-x} \mathrm{Fe}_{x}\right)_{89} \mathrm{Zr}_{7} \mathrm{~B}_{4}$ and $\left(\mathrm{Co}_{1-x} \mathrm{Fe}_{x}\right)_{88} \mathrm{Zr}_{7} \mathrm{~B}_{4} \mathrm{Cu}_{1}{ }^{9,10}$ In this work, we discuss the temperature stability of $K_{U}$ using subsequent thermomagnetic treatments of selected ribbons from our previous reports. The ribbons were initially field annealed at temperatures above or below the primary crystallization temperature, $T_{x 1} \sim 450{ }^{\circ} \mathrm{C}$. Emphasis is placed on the $\left(\mathrm{Co}_{0.975} \mathrm{Fe}_{0.025}\right)_{89} \mathrm{Zr}_{7} \mathrm{~B}_{4}$ composition for which a large value of $K_{U}\left(\sim 20000-25000 \mathrm{ergs} / \mathrm{cm}^{3}\right)$ was observed after field crystallization to produce $\mathrm{BCC}, \mathrm{FCC}$, and $\mathrm{HCP}$ nanocrystals surrounded by an intergranular amorphous phase. ${ }^{9,10,13} \mathrm{Re}-$ sults are also discussed for alloys with varying $\mathrm{Fe}$, Co content and the $\left(\mathrm{Co}_{0.88} \mathrm{Fe}_{0.12}\right)_{79.4} \mathrm{Nb}_{2.6} \mathrm{Si}_{9} \mathrm{~B}_{9}$ composition investigated by other authors. ${ }^{11,12}$

Amorphous ribbons were synthesized by arc-melting and single roller wheel melt spinning. Toroidal wound cores were annealed in flowing $\mathrm{N}_{2}$ for $1 \mathrm{~h}$ with a $2 \mathrm{~T}$ transverse field. The cores were then reannealed with or without a longitudinal applied saturating field of $H>50$ Oe under flowing $\mathrm{N}_{2}$. Dynamic $B-H$ loops at $f=3 \mathrm{kHz}$ were measured using ac permeametry to estimate the anisotropy field, $H_{K}$, by extrapolating the low-field linear portion of the $B-H$ loop to saturation. The room temperature saturation magnetization, $M_{S}$, was measured by vibrating sample magnetometry. $H_{K}$ and $M_{S}$ were used to estimate the value of $K_{U}$ using the expression $K_{U}=\left(H_{K} M_{S}\right) / 2$. Phase identification and microstructural investigations are discussed in detail elsewhere. ${ }^{9,10,13}$

A dramatic difference in the temperature stability of $K_{U}$ was observed for zero field and longitudinal field reannealing of initially transverse field annealed ribbons. This is illustrated in Fig. 1 for a $\left(\mathrm{Co}_{0.975} \mathrm{Fe}_{0.025}\right)_{89} \mathrm{Zr}_{7} \mathrm{~B}_{4}$ alloy after a $T_{\text {anneal }}=350{ }^{\circ} \mathrm{C}$ transverse field annealing treatment (field annealed amorphous). Figure 1(a) shows measured values of $H_{K}$ at room temperature after reannealing the sample at increasing temperatures for $1 \mathrm{~h}$. Because $M_{S}$ of the as-cast and 

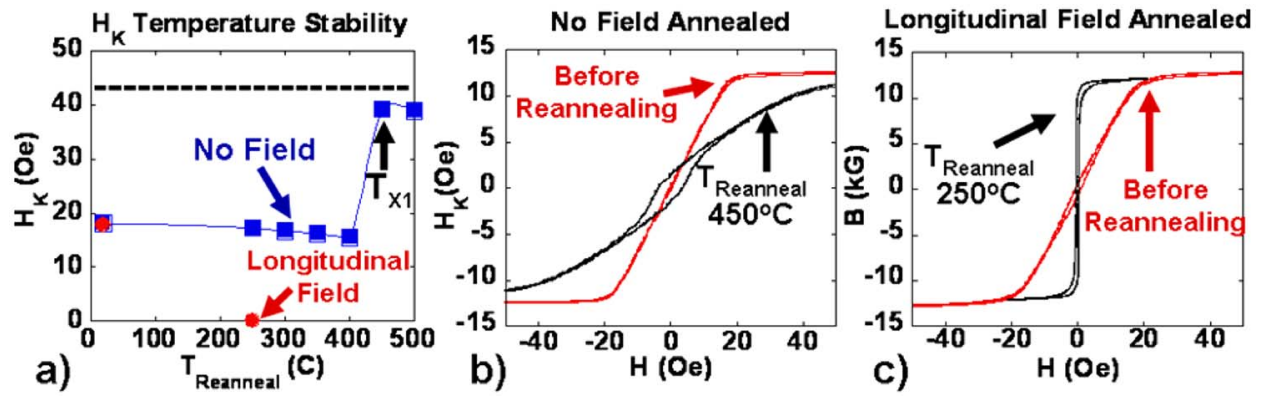

FIG. 1. (Color online) (a) $H_{K}$ measured at room temperature vs reannealing temperature with and without a longitudinal applied field of $H>$ $\sim 50$ Oe for initially transverse field annealed amorphous $\left(T_{\text {anneal }}=350{ }^{\circ} \mathrm{C}\right)$ toroidal $\left(\mathrm{Fe}_{0.025} \mathrm{Co}_{0.975}\right)_{89} \mathrm{Zr}_{7} \mathrm{~B}_{4}$ cores. The dashed line is the measured $H_{K}$ for a transverse field crystallized alloy for comparison $\left(T_{\text {anneal }}=540{ }^{\circ} \mathrm{C}\right)$. Representative $B-H$ loops for the (b) zero-field reannealed and (c) longitudinal field reannealed samples. In (a) $T_{X 1}$ is the reannealing temperature at which the initially amorphous ribbon crystallizes to form a nanocomposite. crystallized ribbons are almost identical for this composition, ${ }^{9,10}$ the trend in $H_{K}$ of Fig. 1 also illustrates the trend in $K_{U}$. For zero-field reannealing treatments, the initial values of $H_{K} \sim 18$ Oe and $K_{U} \sim 8400 \mathrm{ergs} / \mathrm{cm}^{3}$ decrease slightly with increasing reannealing temperatures. An increase occurs upon reannealing at temperatures sufficiently high for crystallization to occur due to the larger values of $H_{K}$ and $K_{U}$ for field crystallized alloys of this composition. ${ }^{9,10}$ This observation demonstrates that the zerofield reannealing treatments are effectively a "self-transverse field anneal" due to the high Curie temperature of the as-cast amorphous ribbon and a transverse remanent domain structure established by the initial transverse field anneal.

Measured $B-H$ loops after the initial transverse field annealing and after zero-field reannealing up to $450{ }^{\circ} \mathrm{C}$ to result in crystallization are presented in Fig. 1(b). The $B-H$ loops are less linear for such "self-transverse field crystallized" alloys as compared to alloys crystallized in a transverse, saturating external field [e.g., Fig. 2(a)] presumably due to the more complex induced anisotropy distribution (e.g., in the vicinity of domain walls). For comparison, the $B-H$ loops are also presented for an initially transverse field annealed amorphous ribbon before and after longitudinal
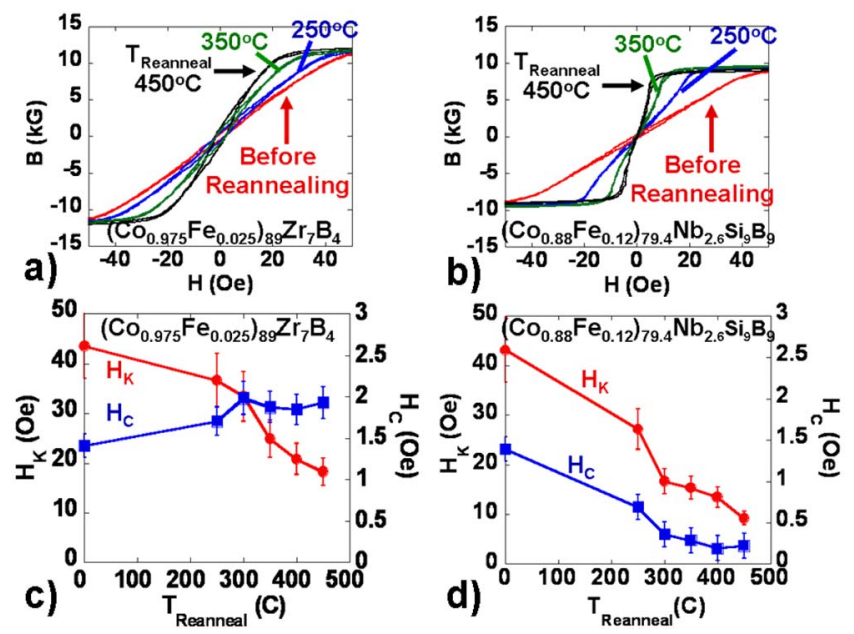

FIG. 2. (Color online) Dynamic $(f=3 \mathrm{kHz}) B-H$ loops measured at room temperature for the high field induced anisotropy alloys (a) $\left(\mathrm{Co}_{0.975} \mathrm{Fe}_{0.025}\right)_{89} \mathrm{Zr}_{7} \mathrm{~B}_{4}$ and (b) $\left(\mathrm{Co}_{0.88} \mathrm{Fe}_{0.12}\right)_{79.4} \mathrm{Nb}_{2.6} \mathrm{Si}_{9} \mathrm{~B}_{9}$ are presented for various longitudinal field reannealing treatments after an initial transverse field crystallization treatment at $T_{\text {anneal }}=540{ }^{\circ} \mathrm{C}$. (c) and (d) show the corresponding values of $H_{K}$ and $H_{C}$ estimated from the $B-H$ loops. field reannealing at $T_{\text {reanneal }}=250{ }^{\circ} \mathrm{C}$ in Fig. 1(c). The transverse field induced anisotropy is completely eliminated even at this low annealing temperature demonstrating that "selffield annealing" effects must be avoided by applying a saturating longitudinal or rotating ${ }^{4}$ magnetic field to properly investigate the temperature stability of $K_{U}$ through reannealing treatments. Therefore we subsequently focus only on results obtained for longitudinal field reannealing treatments.

Figure 2 shows the results of longitudinal field reannealing treatments for the high $K_{U}$ compositions $\left(\mathrm{Co}_{0.975}\right.$ $\left.\mathrm{Fe}_{0.025}\right)_{89} \mathrm{Zr}_{7} \mathrm{~B}_{4}$ [Fig. 2(a)] and $\left(\mathrm{Co}_{0.88} \mathrm{Fe}_{0.12}\right)_{79.4} \mathrm{Nb}_{2.6} \mathrm{Si}_{9} \mathrm{~B}_{9}$ [Fig. 2(b)] after transverse field crystallization at $T_{\text {anneal }}$ $=540{ }^{\circ} \mathrm{C}$. For the field crystallized alloys, $M_{S}$ does not change significantly during reannealing and so $H_{K}$ again represents the trend in $K_{U}$. Both field crystallized alloys exhibit improved temperature stability as compared to the field annealed amorphous alloy of Fig. 1 for which measurable values of $H_{K}$ were eliminated after a longitudinal field reannealing treatment at temperatures as low as $T_{\text {reanneal }}=250{ }^{\circ} \mathrm{C}$. A difference in temperature stability can potentially be explained by a higher atomic mobility in the as-cast amorphous ribbons. ${ }^{2}$ For the $\left(\mathrm{Co}_{0.88} \mathrm{Fe}_{0.12}\right)_{79.4} \mathrm{Nb}_{2.6} \mathrm{Si}_{9} \mathrm{~B}_{9}$ alloy, the $B-H$ loops exhibit a "kink" after longitudinal field reannealing so the estimates of $H_{K}$ are a slight, but consistent, overestimate of the field at which the core saturates.

A correlation between $H_{K}$ and coercivity $\left(H_{C}\right)$ is not observed for the $\left(\mathrm{Co}_{0.975} \mathrm{Fe}_{0.025}\right)_{89} \mathrm{Zr}_{7} \mathrm{~B}_{4}$ alloy [Fig. 2(c)] but a rough correlation is observed for the $\left(\mathrm{Co}_{0.88} \mathrm{Fe}_{0.12}\right)_{79.4}$ $\mathrm{Nb}_{2.6} \mathrm{Si}_{9} \mathrm{~B}_{9}$ alloy [Fig. 2(d)]. A correlation between $H_{K}$ and static values of $H_{C}$ would be reasonable in the framework of the extended random anisotropy model where $K_{U}$ dominates the overall effective anisotropy. ${ }^{5,6}$ However, we cannot address this issue here as the measured values of $H_{C}$ are dynamic and there is some ambiguity in using this model to explain the coercivities of transverse field annealed cores where magnetization occurs primarily through rotation.

In Fig. 3(a), $H_{K}$ is presented as a function of longitudinal field reannealing temperature for several different alloys. Figure 3(b) demonstrates the total reduction in $H_{K}$ after reannealing treatments for $1 \mathrm{~h}$ while Fig. 3(c) presents the fractional reduction in $H_{K}$. These results illustrate that, with the exception of the $x=0.15$ alloy, all of the field crystallized ribbons exhibit enhanced temperature stability of $K_{U}$ as compared to the field annealed amorphous ribbon of Fig. 1. The 

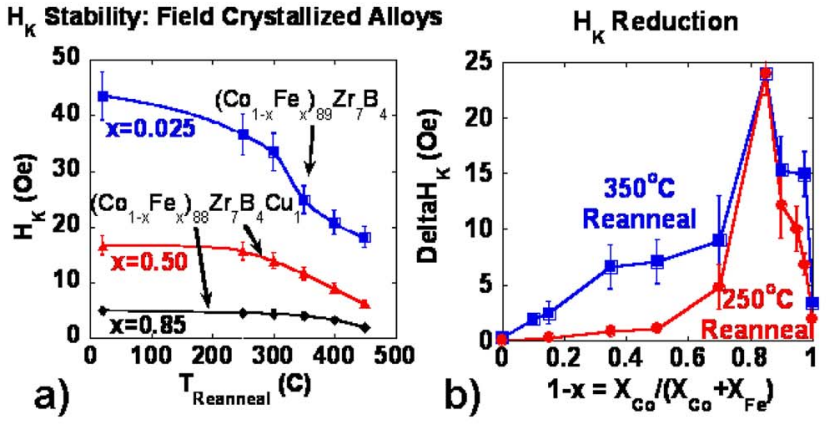

relatively low temperature stability demonstrated by the $\left(\mathrm{Co}_{0.85} \mathrm{Fe}_{0.15}\right)_{89} \mathrm{Zr}_{7} \mathrm{~B}_{4} \mathrm{Cu}_{1}(x=0.15)$ field crystallized alloy is not understood at this time.

The temperature stability of $K_{U}$ can provide information about the potential mechanisms responsible for the field induced anisotropy. Further investigation of the temperature stability of $K_{U}$ is worthwhile in an attempt to clarify the origin of field induced anisotropy in the complex nanocomposites. A comparison between the relative stability of $K_{U}$ for alloys in which different mechanisms of field induced anisotropy are thought to be dominant could be particularly enlightening.

A final set of experiments demonstrate the importance of applying a magnetic field during crystallization to generate the largest $K_{U}$. B-H loops were compared for ribbons crystallized in a transverse field $\left(T_{\text {anneal }}=540{ }^{\circ} \mathrm{C}\right.$ for $\left.1 \mathrm{~h}\right)$ with those obtained for ribbons crystallized without a field and then subsequently reannealed in a transverse field (same temperature and time). In Fig. 4(a), $B-H$ loops measured for the zero-field crystallized $\left(\mathrm{Co}_{0.975} \mathrm{Fe}_{0.025}\right)_{89} \mathrm{Zr}_{7} \mathrm{~B}_{4}$ ribbon are presented and compared to the transverse field crystallized core. No measurable $H_{K}$ is found after transverse field reannealing of the zero-field crystallized ribbon but there is a large $H_{K}$ $\sim 43$ Oe $\left(K_{U} \sim 22000 \mathrm{ergs} / \mathrm{cm}^{3}\right)$ for the transverse field crystallized ribbon. Similar results have been obtained for other Co:Fe ratios (for example, $x=0.00, x=0.50$, and $x$ $=1.00$ ) and in all cases transverse field crystallization treatments are more effective than transverse field reannealing of zero-field crystallized ribbons. For the high $K_{U}\left(\mathrm{Co}_{0.88}\right.$ $\left.\mathrm{Fe}_{0.12}\right)_{79.4} \mathrm{Nb}_{2.6} \mathrm{Si}_{9} \mathrm{~B}_{9}$ alloy a measurable $H_{K} \sim 22$ Oe $\left(K_{U}\right.$

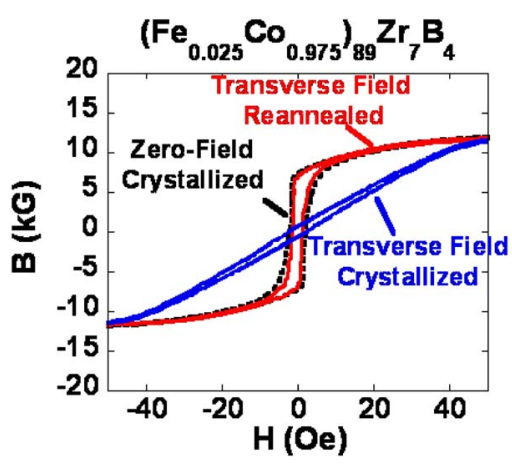

FIG. 4. (Color online) Dynamic $B-H$ loops for a $\left(\mathrm{Co}_{0.975} \mathrm{Fe}_{0.025}\right)_{89} \mathrm{Zr}_{7} \mathrm{~B}_{4}$ alloy after zero-field crystallization (dashed, black) at $T_{\text {anneal }}=540{ }^{\circ} \mathrm{C}$ followed by a transverse field reannealing treatment (solid, red) under the same annealing conditions and compared to a transverse field crystallized ribbon at $T_{\text {anneal }}=540^{\circ} \mathrm{C}$ (solid, blue).

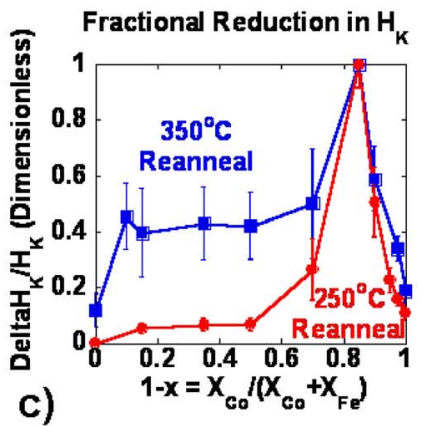

FIG. 3. (Color online) (a) Measured values of $H_{K}$ at room temperature for several $\left(\mathrm{Co}_{1-x} \mathrm{Fe}_{x}\right)_{89} \mathrm{Zr}_{7} \mathrm{~B}_{4}$ and $\left(\mathrm{Co}_{1-x}\right.$ $\left.\mathrm{Fe}_{x}\right)_{88} \mathrm{Zr}_{7} \mathrm{~B}_{4} \mathrm{Cu}_{1}$ alloys after heating to each reannealing temperature indicated for $1 \mathrm{~h}$ with a longitudinal applied field of $H>\sim 50$ Oe and then cooling to room temperature. (b) The reduction in $H_{K}$ and (c) the fractional reduction in $H_{K}$ for field crystallized alloys as a function of composition after a longitudinal field reannealing treatment at $T=250{ }^{\circ} \mathrm{C}$ or $T=350{ }^{\circ} \mathrm{C}$. $\sim 9000 \mathrm{ergs} / \mathrm{cm}^{3}$ ) was induced by transverse field reannealing treatment but it was lower than the $H_{K} \sim 43$ Oe $\left(K_{U}\right.$ $\sim 17500 \mathrm{ergs} / \mathrm{cm}^{3}$ ) obtained after transverse field crystallization. We suggest that these observations are likely due to a higher atomic mobility during the early stages of primary crystallization.

Temperature stability of field induced anisotropy, $K_{U}$, in amorphous and nanocrystalline/amorphous nanocomposite alloys was investigated. We conclude that (1) field annealing treatments are necessary to investigate the temperature stability of $K_{U}$, (2) $K_{U}$ of field crystallized alloys typically exhibit higher temperature stability than alloys remaining amorphous after field annealing, and (3) larger $K_{U}$ is obtained for alloys crystallized in a field rather than reannealed in a field after zero-field crystallization. Field crystallization may be required for elevated temperature applications when field induced anisotropy is critical for performance. Experiments examining the temperature stability of $K_{U}$ for field crystallized alloys deserve further investigation as they may help provide insight into the mechanistic origin of $K_{U}$ in these complex systems.

P.R.O. acknowledges support from the National Defense Science and Engineering Graduate Research Fellowship throughout the preparation of this manuscript. Funding from the National Science Foundation is also acknowledged (NSF Grant No. DMR-0406220).

${ }^{1}$ S. Chikazumi, Physics of Ferromagnetism (Wiley, New York, 1986).

${ }^{2}$ R. C. O'Handley, Modern Magnetic Materials: Principles and Applications (Wiley Interscience, New York, 1999).

${ }^{3}$ G. Herzer, IEEE Trans. Magn. 26, 1397 (1990).

${ }^{4}$ K. Suzuki, N. Ito, J. S. Garitaonandia, and J. D. Cashion, J. Appl. Phys. 99, 08F114 (2006).

${ }^{5} \mathrm{~K}$. Suzuki and G. Herzer, in Advanced Magnetic Nanostructures, edited by D. Sellmyer and R. Skomski (Springer, New York, 2006), pp. 365-401.

${ }^{6}$ K. Suzuki, N. Ito, S. Saranu, U. Herr, A. Michels, and J. S. Garitaonandia, J. Appl. Phys. 103, 07E730 (2008).

${ }^{7}$ J. McCord and J. Paul, IEEE Trans. Magn. 39, 2359 (2003).

${ }^{8} \mathrm{R}$. Hasegawa, in Properties and Applications of Nanocrystalline Alloys from Amorphous Precursors, edited by B. Idzikowski, P. Svec, and M. Miglierini (Kluwer Academic Publishers, Dordrecht, The Netherlands, 2005), pp. 189-207.

${ }^{9}$ P. R. Ohodnicki, D. E. Laughlin, M. E. McHenry, V. Keylin, and J. Huth J. Appl. Phys. 104, 113909 (2009).

${ }^{10}$ P. R. Ohodnicki, Ph.D. thesis, Carnegie Mellon University, 2008.

${ }^{11}$ S. F. Y. Yoshizawa, D. H. Ping, M. Ohnuma, and K. Hono, Scr. Mater. 48, 863 (2003)

${ }^{12}$ S. F. Y. Yoshizawa, D. H. Ping, M. Ohnuma, and K. Hono, Mater. Sci. Eng., A 375-377, 207 (2004).

${ }^{13}$ P. R. Ohodnicki, Y. L. Qin, D. E. Laughlin, M. E. McHenry, M. Kodzuka, T. Ohkubo, K. Hono, and M. A. Willard, Acta Mater. 57, 87 (2009). 